\title{
Linear best method for recovering the second derivatives of Hardy class functions
}

\author{
Mikhail Ovchintsev $^{1 *}$ \\ ${ }^{1}$ Moscow State University of Civil Engineering, Yaroslavskoye shosse, 26, Moscow, 129337, Russia
}

\begin{abstract}
The linear best method for approximating the second derivatives of Hardy class functions defined in the unit circle at zero in accordance with the information about their values in a finite number of points forming a regular polygon is found. The paper is divided into three sections. The first contains the necessary concepts and results from the work of K.Yu. Osipenko. It also recalls some results obtained by S. Ya. Havinson and other authors. In the second section, the error of the best method is calculated, and the corresponding extremal functions are written out. The third proves that the linear best approximation method is unique, and its coefficients are calculated.
\end{abstract}

\section{Introduction}

Let $\mathrm{W}$ be some set lying in a linear complex space $\mathrm{X}$ and $L, l_{1}, \ldots, l_{n}-$ linear complex functionals defined on X. If $S\left(t_{1}, \ldots, t_{n}\right)$ is a complex function of $\mathrm{n}$ complex variables, then the approximation error of the functional $\mathrm{L}$ by the values of the functionals $l_{1}, \ldots, l_{n}$ by the method $\mathrm{S}$ on the set $\mathrm{W}$ is the quantity

$$
r_{n}(S)=\sup _{x \in W} \mid L(x)-S\left(l_{1}(x), \ldots, l_{n}(x) \mid .\right.
$$

Complex function $S_{0}\left(t_{1}, \ldots, t_{n}\right)$ is called the best approximation method if

$$
r_{n}\left(S_{0}\right)=\inf _{S} r_{n}(s)
$$

The existence of the linear best method was proved in [1]

$$
S_{0}=\sum_{k=1}^{n} c_{k} l_{k}(x)
$$

(under certain conditions on the set W). In addition, it was found that the error of the best approximation method can be calculated by the following formula

$$
r_{n}\left(S_{0}\right)=\sup _{\substack{x \in W \\ l_{1}(x)=. .=l_{n}(x)}}|L(x)| .
$$

Let's denote the unit circle by $=\{z:|z|<1\}$, and the unit circumference by $G=$

${ }^{*}$ Corresponding author: 6714543@rambler.ru 
$\{z:|z|=1\}$. Let

$$
H_{p}^{1}=\left\{f(z), f(z) \in H^{p}: \int_{\Gamma}|f(\zeta)|^{p} d \varphi \leq 1\right\}-
$$

Be the unit ball in the Hardy space $(1 \leq p<\infty)$ (see the definition of Hardy classes in [2], [3]). We note that the problems of optimal recovery of functions belonging to certain classes and derivatives of functions with respect to their values in a finite number of points have been studied in many papers (see, for example, [1], [4-12]). This work is a continuation of [13].

Let's consider the following system of points forming a regular polygon

$$
z_{1}=R, R e^{i \frac{2 \pi}{n}}, \ldots, z_{n}=R e^{i(n-1) \frac{2 \pi}{n}},
$$

where $R$ - set number; $0<R<1$.

Let's denote by

$$
L(f)=f^{\prime \prime}(0), \quad l_{1}(f)=f\left(z_{1}\right), \ldots, l_{n}(f)=f\left(z_{n}\right)
$$

a system of linear functionals defined in Hardy space $H_{p}$, where $1 \leq p<\infty$. Then the error of the best approximation method (we denote it by $r_{2, p}\left(0, z_{1}, \ldots, z_{n}\right)$ ) can be calculated by the formula (see (1))

$$
r_{2, p}\left(0, z_{1}, \ldots, z_{n}\right)=\sup _{\substack{f \in H_{p}^{1} \\ f\left(z_{1}\right)=. .=f\left(z_{n}\right)=0}}\left|f^{\prime \prime}(0)\right| .
$$

Recall some results from [14] (see also [15]). Let $\omega(\zeta)$ be a complex function bounded on the circumference $\mathrm{G}$. Then the following duality relation holds

$$
\sup _{f \in H_{1}^{1}}\left|\int_{G} f(\zeta) \omega(\zeta) d \zeta\right|=\min _{\varphi \in B(K)} \operatorname{vraima}|\omega(\zeta)-\varphi(\zeta)|,(4)
$$

where $B(K)$ - the set of all bounded analytic functions in K. Moreover, the functions $f^{*}(z) \in H_{1}^{1}, \varphi^{*}(z) \in B(K)$ are extremal in equality (4) if and only if the following equality holds almost everywhere on the circumference $\mathrm{G}$

$$
f^{*}(\zeta)\left[\omega(\zeta)-\varphi^{*}(\zeta)\right] d \zeta=e^{i \delta} \lambda\left|f^{*}(\zeta)\right| d \varphi,
$$

where $\delta \in R$, and $\lambda$ - the total value in the left and right side of equality (4).

If now $p>1$. And if $\omega(\zeta) \in L_{q}(\mathrm{G})$ (here

$\left.\frac{1}{p}+\frac{1}{q}=1\right)$, then the following duality relation holds

$$
\sup _{f \in H_{p}^{1}}\left|\int_{\Gamma} f(\zeta) \omega(\zeta) d \zeta\right|=\min _{\varphi \in H_{q}}\left\{\int_{\Gamma}|\omega(\zeta)-\varphi(\zeta)|^{q} d \varphi\right\}^{\frac{1}{q}} .
$$

The extremal function $\varphi^{*}(\zeta)$ is unique on the right side of equality (4) and on the right side of equality (6). The extreme function $f^{*}(z)$ on the left side of equality (6) is unique up to a constant factor $e^{i \delta} \quad(\delta \in R)$. In addition, in order for the functions $f^{*}(z) \in H_{p}^{1} \quad$ и $\varphi^{*}(z) \in H_{p}$ to be extremal in equality (6), it is necessary and sufficient that the following 
relation holds almost everywhere on the boundary G:

$$
f^{*}(\zeta)\left[\omega(\zeta)-\varphi^{*}(\zeta)\right] d \zeta=e^{i \alpha} \lambda\left|f^{*}(\zeta)\right|^{p} d \varphi,
$$

where $\lambda-$ the total value in equality (6), $\alpha \in R$. Recall that the extreme function $f^{*}(z)$ satisfies the condition (for all $1 \leq p<\infty$ )

$$
\int_{\mathrm{G}}\left|f^{*}(\zeta)\right|^{p} d \varphi=1 .
$$

Further, if $\omega(\zeta)$ is the boundary value on the circumference $G$ of the function $\omega(z)$ meromorphic in the circle $\mathrm{K}$ with poles $\beta_{1}, \ldots, \beta_{m}$ (each pole is repeated as many times as its multiplicity is), then the product

$$
R(z)=f^{*}(z)\left[\omega(z)-\varphi^{*}(z)\right]
$$

analytically on the boundary $\mathrm{G}$ and has in $\mathrm{K}$

$$
T=m-1
$$

zeros. It is obtained in [14]-[15]

$$
R(z)=C \frac{\prod_{k=1}^{m-1}\left(z-\alpha_{k}\right)\left(1-\overline{\alpha_{k}} z\right)}{\prod_{k=1}^{m}\left(z-\beta_{k}\right)\left(1-\overline{\beta_{k}} z\right)}
$$

where $\left|\alpha_{k}\right| \leq 1, k=1, \ldots, m-1 ; C-$ constant number.

We give some formulas that we will use later (see [13]). Let's denote by

$$
B(z)=\prod_{k=1}^{n} \frac{z-z_{k}}{1-\overline{z_{k}} z}-
$$

the final Blaschke product, in which the points $z_{1}, \ldots, z_{n}$ are of the form (2). Then the following equalities hold

$$
\begin{aligned}
& B(0)=-R^{n}, \\
& B^{(j)}(0)=0,
\end{aligned}
$$
that

where $1 \leq j \leq n-1$. In addition, it was established in [13] (see [13], formula (42))

$$
B^{\prime}\left(z_{k}\right)=e^{-i(k-1) \frac{2 \pi}{n}} \frac{R^{n-1} n}{1-R^{2 n}}
$$

where $k=1, \ldots, n$.

\section{Best recovery methods}

\subsection{Finding the best method error}

Lemma 1. The following relation holds:

$$
d=\sup _{g \in H_{p}^{1}}\left|g^{\prime \prime}(0)\right|=\frac{2^{\frac{1}{q}}}{\pi^{\frac{1}{p}} .}
$$

Moreover, if $p>1$, then the extremal function $g^{*}(z)$ of problem (15) is unique up to a 
constant factor $e^{i \delta} \quad(\delta \in R)$ and has the form

$$
g^{*}(z)=e^{i \delta} \frac{1}{(2 \pi)^{\frac{1}{p}}} z^{2} .
$$

In the case when $p=1$, the extremal function of problem (15) is not unique. Any of the extremal functions of problem (15) with $p=1$ has the form

$$
g^{*}(z)=e^{i \delta} \frac{(z-a)(1-\bar{a} z)(z-b)(1-\bar{b} z)}{2 \pi\left(\left(1+|a|^{2}\right)\left(1+|b|^{2}\right)+\bar{a} b+a \bar{b}\right)},
$$

where $\delta \in R ; a, b-$ any complex numbers satisfying the conditions:

$$
|a| \leq 1,|b| \leq 1
$$

Proof. Since $g^{*}(z)=\frac{1}{(2 \pi)^{\frac{1}{p}}} z^{2} \in H_{p}^{1}$, then

$$
d \geq \frac{2^{\frac{1}{q}}}{\pi^{\frac{1}{p}}}
$$

For all $1 \leq p<\infty$. After this, we consider separately two cases: the case when $p>1$ and when $p=1$.

First, $p>1$ and $g(z)-$ any function belonging to the unit ball $H_{p}^{1}$. Then

$$
\left|g^{\prime \prime}(0)\right|=\left|\frac{2}{2 \pi i} \int_{\mathrm{G}} \frac{g(\zeta)}{\zeta^{3}} d \zeta\right| \leq \frac{1}{\pi} \int_{\mathrm{G}}|g(\zeta)| d \varphi \leq \frac{1}{\pi}\left(\int_{\mathrm{G}}|g(\zeta)|^{p} d \varphi\right)^{\frac{1}{p}}\left(\int_{\mathrm{G}} d \varphi\right)^{\frac{1}{q}} \leq \frac{1}{\pi}(2 \pi)^{\frac{1}{q}}=\frac{2^{\frac{1}{q}}}{\pi^{\frac{1}{p}}} .
$$

This (see (18)) implies equality (15). The extremal function of problem (15) is unique for $p>1$ (up to a factor equal to unity in modulo) and has the form (16) (see (19) and the introduction).

Let's assume now that $p=1$. In this case (see (15)), $d=\frac{1}{\pi}$. Let $g^{*}(z)$ be any extreme function of problem (15) for $p=1$ (if there are several) and let $\varphi^{*}(z)$ be the extremal function on the right side of duality relation (4) for the corresponding function equal to (see (19))

$$
\omega(\zeta)=\frac{1}{\pi i} \frac{1}{\zeta^{3}}
$$

then (see (5)) these extremal functions satisfy the relation

$$
g^{*}(\zeta)\left[\frac{2}{2 \pi i} \frac{1}{\zeta^{3}}-\varphi^{*}(\zeta)\right] d \zeta=e^{i \delta} \lambda\left|g^{*}(\zeta)\right| d \varphi .
$$

Since $d \zeta=i \zeta d \varphi$, it's easy to verify that the functions

$$
g^{*}(z)=\frac{1}{\pi} z^{2}, \varphi^{*}(z)=0, \lambda=\frac{1}{\pi}, e^{i \delta}=1
$$

satisfy relation $(20)$. Hence, $\varphi^{*}(z)=0(z \in \bar{K})$. It follows that any of the extremal functions $g^{*}(z)$ of problem (15) for $p=1$ satisfies the equation (see (9), (11)) 


$$
g^{*}(z) \frac{1}{\pi i} \frac{1}{z^{3}}=C_{1} \frac{(z-a)(1-\bar{a} z)(z-b)(1-\bar{b} z)}{z^{3}},
$$

where $C_{1}$ - some constant number; $|a| \leq 1,|b| \leq 1$. Therefore,

$$
g^{*}(z)=C(z-a)(1-\bar{a} z)(z-b)(1-\bar{b} z) \text {. }
$$

Let's find constant number $\mathrm{C}\left(C=\pi i C_{1}\right)$. To do this, it is necessary to calculate the following integral (see (8))

$$
\int_{\mathrm{G}}|\zeta-a||1-\bar{a} \zeta||\zeta-b||1-\overline{b \zeta}| d \varphi .
$$

Let the point $\zeta \in G$, i.e. let $|\zeta|=1$. Then

$$
d \varphi=\frac{d \zeta}{i \zeta}, \bar{\zeta}=\frac{1}{\zeta},|\zeta-a|=|1-\bar{a} \zeta|,|\zeta-b|=|1-\bar{b} \zeta| .
$$

From here we obtain

$$
\begin{aligned}
\int_{\mathrm{G}}|\zeta-a||1-\bar{a} \zeta| \mid \zeta- & b \| 1-\bar{b} \zeta\left|d \varphi=\int_{\mathrm{G}}\right| \zeta-\left.a\right|^{2}|\zeta-b|^{2} d \varphi \\
& =\frac{1}{i} \int_{\mathrm{G}}(\zeta-a)(\bar{\zeta}-\bar{a})(\zeta-b)(\bar{\zeta}-\bar{b}) \frac{d \zeta}{\zeta} \\
& =\frac{1}{i} \int_{\mathrm{G}}(\zeta-a)\left(\frac{1}{\zeta}-\bar{a}\right)(\zeta-b)\left(\frac{1}{\zeta}-\bar{b}\right) \frac{d \zeta}{\zeta}=\frac{1}{i} \int_{\mathrm{G}} \frac{\left(\bar{a} b+\left(1+|a|^{2}\right)\left(1+|b|^{2}\right)+a \bar{b}\right) d \zeta}{\zeta} \\
& =2 \pi\left(\left(1+|a|^{2}\right)\left(1+|b|^{2}\right)+\bar{a} b+a \bar{b}\right) .
\end{aligned}
$$

Therefore (see (8))

$$
|C|=\frac{1}{2 \pi\left(\left(1+|a|^{2}\right)\left(1+|b|^{2}\right)+\bar{a} b+a \bar{b}\right)} .
$$

Therefore, the extremal function of problem (15) in the case when $p=1$ has the form (17) (see (21)). Conversely, it is easy to verify that a function of the form (17) is an extremal function of problem (15) for $p=1$. The lemma is proved.

Theorem 1. The error of the best method for approximating the values of $f^{\prime \prime}(0)$ from functions belonging to the unit ball $H_{p}^{1}$ by their values at points $z_{1}, \ldots, z_{n}$ (of the form (2); $n \geq 3$ ) can be calculated by the formula

$$
r_{2, p}\left(0, z_{1}, \ldots, z_{n}\right)=R^{n} \frac{2^{\frac{1}{q}}}{\pi^{\frac{1}{p}}}
$$

In addition, if $p>1$ extremal function $f^{*}(z)$ of problem (3) is unique up to a factor $e^{i \delta}$, where $\delta-$ constant real number and has the form

$$
f^{*}(z)=e^{i \delta} \frac{2^{\frac{1}{q}}}{\pi^{\frac{1}{p}}} z^{2} B(z)
$$

If $p=1$, then there are infinitely many extremal functions of problem (3) and any of them has the form 


$$
f^{*}(z)=e^{i \delta} \frac{(z-a)(1-\bar{a} z)(z-b)(1-\bar{b} z)}{2 \pi\left(\left(1+|a|^{2}\right)\left(1+|b|^{2}\right)+\bar{a} b+a \bar{b}\right)} B(z),
$$

where $a, b-$ any complex numbers satisfying the conditions $|a| \leq 1,|b| \leq 1 ; \delta \in R$.

Proof. Let's denote by

$$
E=\left\{f(z): f(z) \in H_{p}^{1}, f\left(z_{1}\right)=. .=f\left(z_{n}\right)=0\right\}
$$

the family of analytic functions in the circle K. First, we factorize the family of functions E. Let $f(z) \in E$. Denote (see (25))

$$
g(z)=\frac{f(z)}{B(z)}
$$

It is obvious that

$$
f(z)=B(z) g(z),
$$

where $g(z) \in H_{p}^{1}$. Conversely, any of the functions $\mathrm{f}(\mathrm{z})$ having the form (26) belongs to the family E. Since (see (12), (13))

$$
f^{\prime \prime}(0)=B(0) g^{\prime \prime}(0)=-R^{n} g^{\prime \prime}(0)
$$

then it follows that (see (15))

$$
r_{2, p}\left(0, z_{1}, \ldots, z_{n}\right)=R^{n} \sup _{g \in H_{p}^{1}}\left|g^{\prime \prime}(0)\right|=R^{n} \frac{2^{\frac{1}{q}}}{\pi^{\frac{1}{p}}} .
$$

It is clear that the extremal function of problem (3) for $p>1$ has the form (23), and for $p=1$, any of the extremal functions of problem (3) has the form (24).

\subsection{Finding the coefficients of the linear best approximation method}

Let $\sum_{k=1}^{n} c_{k} f\left(z_{k}\right)$ - linear best method of recovering the value of $f^{\prime \prime}(0)$ by the values of $f\left(z_{1}\right), \ldots, f\left(z_{n}\right)$, where $f(z) \in H_{p}^{1}$, and points $z_{1}, \ldots, z_{n}$ has the form $(2)$, and $f^{*}(z)$ is an extremal function of problem (3). Then, it is easy to verify that $f^{*}(z)$ is an extremal function of the problem

$$
\sup _{f \in H_{p}^{1}}\left|f^{\prime \prime}(0)-\sum_{k=1}^{n} c_{k} f\left(z_{k}\right)\right|=r_{2, p}\left(0, z_{1}, \ldots, z_{n}\right) .
$$

Theorem 2. Let points $z_{1}, \ldots, z_{n}$ have the form (2) $(n \geq 3)$. Then the linear best method $\sum_{k=1}^{n} c_{k} f\left(z_{k}\right)$ of recovering $f^{\prime \prime}(0)$ by values of functions at points $z_{1}, \ldots, z_{n}$ is unique $\left(f(z) \in H_{p} ; 1 \leq p<\infty\right)$, and its coefficients are found by the formulas

$$
c_{k}=\frac{2}{n R^{2}}\left(1-R^{2 n}\right) e^{-i(k-1) \frac{2 \pi}{n}}
$$

for all values of $k=1, \ldots, n$.

Proof. Let $f(z)$ be any function belonging to the unit ball $H_{p}^{1}(1 \leq p<\infty)$. Let's consider the following integral

$$
J=\frac{1}{\pi i} \int_{\mathrm{G}} \frac{B(0)}{B(\zeta) \zeta^{3}} f(\zeta) d \zeta
$$


Let's estimate the integral $\mathrm{J}$ in modulo (see (12), (22))

$|J| \leq \frac{1}{\pi} \int_{\mathrm{G}}\left|\frac{B(0)}{B(\zeta) \zeta^{3}}\right||f(\zeta)| d \varphi=\frac{R^{n}}{\pi} \int_{\mathrm{G}}|f(\zeta)| d \varphi \leq \frac{R^{n}}{\pi}\left(\int_{\mathrm{G}}|f(\zeta)|^{p} d \varphi\right)^{\frac{1}{p}}\left(\int_{\mathrm{G}} d \varphi\right)^{\frac{1}{q}} \leq \frac{2^{\frac{1}{q}} R^{n}}{\pi^{\frac{1}{p}}}=r_{2, p}\left(0, z_{1}, \ldots, z_{n}\right)$.

Let $p=1$. Then (see (22))

$$
|J| \leq \frac{1}{\pi} \int_{\mathrm{G}}|B(0)||f(\zeta)| d \varphi \leq \frac{R^{n}}{\pi}=r_{2,1}\left(0, z_{1}, \ldots, z_{n}\right) .
$$

Now we calculate the integral J. Denote

$$
D(z)=\frac{B(0)}{B(z) z^{3}} .
$$

Function $\mathrm{D}(\mathrm{z})$ has singular points (poles) at points $0, z_{1}, \ldots, z_{n}$. Then the function $\mathrm{D}(\mathrm{z})$ can be represented as

$$
D(z)=\frac{c_{-3}}{z^{3}}+\frac{c_{-2}}{z^{2}}+\frac{c_{-1}}{z}-\frac{a_{1}}{z-z_{1}}-\cdots-\frac{a_{n}}{z-z_{n}}+v(z),
$$

Where $v(z)$ - analytic function in the circle $\mathrm{K} ; c_{-3}, c_{-2}, c_{-1}, a_{1}, \ldots, a_{n}-$ constant numbers. Let's find these numbers. It is obvious that (see (32), (13))

$$
\begin{gathered}
c_{-3}=\lim _{z \rightarrow 0} z^{3} D(z)=\lim _{z \rightarrow 0} \frac{B(0)}{B(z)}=1, \\
\mathrm{c}_{-2}=\lim _{z \rightarrow 0}\left(\frac{B(0)}{B(z)}\right)^{\prime}=B(0) \lim _{z \rightarrow 0} \frac{-B^{\prime}(z)}{B^{2}(z)}=0, \\
c_{-1}=-B(0) \lim _{z \rightarrow 0}\left(\frac{B^{\prime}(z)}{B^{2}(z)}\right)^{\prime}=-B(0) \lim _{z \rightarrow 0} \frac{B^{\prime \prime}(z) B^{2}(z)-2 B(z)\left(\left(B^{\prime}(z)\right)^{2}\right)^{\prime}}{B^{4}(z)}=0
\end{gathered}
$$

After that, we calculate the deductions at simple poles $z_{1}, \ldots, z_{n}$. We have (see (14))

$$
-a_{k}=\underset{z=z_{k}}{\operatorname{res}} D(z)=\lim _{z \rightarrow z_{k}}\left(z-z_{k}\right) \frac{B(0)}{B(z) z^{3}}=\frac{B(0)}{B^{\prime}\left(z_{k}\right) z_{k}^{3}}=\frac{-R^{n}\left(1-R^{2 n}\right)}{R^{n-1} n R^{3} e^{i 2(k-1) \frac{2 \pi}{n}}} e^{i(k-1) \frac{2 \pi}{n}}=-\frac{1}{n R^{2}}\left(1-R^{2 n}\right) e^{-i(k-1) \frac{2 \pi}{n}}
$$

Therefore,

$$
J=\frac{2}{2 \pi i} \int_{\mathrm{G}}\left(\frac{1}{\zeta^{3}}-\frac{a_{1}}{\zeta-z_{1}}-\cdots-\frac{a_{n}}{\zeta-z_{n}}+v(z)\right) f(\zeta) d \zeta=f^{\prime \prime}(0)-\sum_{k=1}^{n} 2 a_{k} f\left(z_{k}\right) .
$$
(31))

It follows that if $f(z)$ - any function belonging to the unit ball $H_{p}^{1}$, then (see (30),

$$
\left|f^{\prime \prime}(0)-\sum_{k=1}^{n} 2 a_{k} f\left(z_{k}\right)\right| \leq r_{2, p}\left(0, z_{1}, \ldots, z_{n}\right) .
$$

Thus, the method $\sum_{k=1}^{n} 2 a_{k} f\left(z_{k}\right)$ is the linear best approximation method. From here, $c_{k}=2 a_{k} \quad(\mathrm{k}=1, \ldots, \mathrm{n})$ are the coefficients of the linear best approximation method and are found by formulas (28).

We note that (see (28))

$$
c_{k} \neq 0
$$


for all $k=1, \ldots, n$. Let's consider the linear best approximation method $\sum_{k=1}^{n} c_{k} f\left(z_{k}\right)$, in which the coefficients $c_{k}$ are calculated by the formulas (28) $(\mathrm{k}=1, \ldots, \mathrm{n})$. Then

$$
\sup _{f \in H_{p}^{1}}\left|\int_{G} \omega(\zeta) f(\zeta) d \zeta\right|=r_{2, p}\left(0, z_{1}, \ldots, z_{n}\right)
$$

where

$$
\omega(\zeta)=\frac{1}{2 \pi i}\left(\frac{2}{\zeta^{3}}-\sum_{k=1}^{n} \frac{c_{k}}{\zeta-z_{k}}\right) .
$$

and, therefore, for all values $1 \leq p<\infty$ the following relation holds (see (5), (7), (27))

$$
f^{*}(\zeta)\left(\omega(\zeta)-\varphi^{*}(\zeta)\right) d \zeta=e^{i \delta_{1}} \lambda\left|f^{*}(\zeta)\right|^{p} d \varphi,
$$

where $f^{*}(\zeta)$ - extremal function of problem $(3), \varphi^{*}(\zeta)$ - extremal function on the right side of the dual extremal problem (see (4), (6)), $\delta_{1}-$ real constant, $\lambda=$ $r_{2, p}\left(0, z_{1}, \ldots, z_{n}\right)$. Note that function $R(z)=f^{*}(z)\left[\omega(z)-\varphi^{*}(z)\right]$ does not have zeros in the circle K (see (23), (24), (10); when applying formula (24), we must assume $a=b=0$ ).

Let's suppose there is another linear best approximation method $\sum_{k=1}^{n} \widetilde{c_{k}} f\left(z_{k}\right)$.

Then the following relation holds

$$
f^{*}(\zeta)\left(\frac{1}{2 \pi i}\left(\frac{2}{\zeta^{3}}-\sum_{k=1}^{n} \frac{\widetilde{c_{k}}}{\zeta-z_{k}}\right)-\varphi_{1}^{*}(\zeta)\right) d \zeta=e^{i \delta_{2}} \lambda\left|f^{*}(\zeta)\right|^{p} d \varphi,
$$

where $\varphi_{1}^{*}-$ the extremal function on the right side in equality (6) (or in equality (4)) for the corresponding function $\omega(\zeta) ; \delta_{2}-$ real number. Let's consider the following function:

$$
W(z)=\frac{\frac{1}{2 \pi i}\left(\frac{2}{z^{3}}-\sum_{k=1}^{n} \frac{\widetilde{c_{k}}}{z-z_{k}}\right)-\varphi_{1}^{*}(z)}{\frac{1}{2 \pi i}\left(\frac{2}{z^{3}}-\sum_{k=1}^{n} \frac{c_{k}}{z-z_{k}}\right)-\varphi^{*}(z)} .
$$

Since (see. (38))

$$
\lim _{z \rightarrow 0} W(z)=1, \lim _{z \rightarrow z_{k}} W(z)=\frac{\widetilde{c_{k}}}{c_{k}} \quad(\mathrm{k}=1, \ldots, \mathrm{n}),
$$

then $W(z)-$ function analytical in $\bar{K}$. Since relations (40) and (41) hold, then $W(\zeta)=e^{i \delta} \quad\left(\zeta \in \mathrm{G} ; \delta=\delta_{2}-\delta_{1}\right)$. Since $\mathrm{W}(0)=1$, then $W(z)=1$ for all values of $z \in$ $\bar{K}$. It follows from this (see (42)) $\breve{c_{k}}=c_{k}$ for all values of $k=1, \ldots, n$. Thus, for all values $1 \leq p<\infty$ the linear best approximation method is unique. The theorem is proved.

\section{Results}

The error of the best method for approximating the values of $f^{\prime \prime}(0)$ from functions of the class $H_{p}, 1 \leq p<\infty$ by their values $\mathrm{f} f\left(z_{1}\right), \ldots, f\left(z_{n}\right)$, where points $z_{1}, \ldots, z_{n}$ have the form (2) are obtained in this study. The corresponding extreme functions are written out. It is established that if $1<p<\infty$, these extreme functions are unique up to a constant factor $e^{i \delta}(\delta \in R)$. If $p=1$, then the extremal functions are not unique. The type of all such functions is written out. It is proved that the linear best approximation method is unique 
(for all $1 \leq p<\infty$ ), and all its coefficients are calculated.

\section{Conclusion}

Thus, the problem of the optimal recovery of the second derivatives of the functions of Hardy class at zero by their values in a finite number of points forming a regular polygon centered at zero is solved. It would be interesting to solve the problem of optimal recovery of derivatives $f^{(N)}(0)$, where $N-$ any natural number by values $f\left(z_{1}\right), \ldots, f\left(z_{n}\right)$ $\left(f(z) \in H_{p}\right)$, and points $z_{1}, \ldots, z_{n}$ form a regular polygon.

\section{References}

1. K.Yu. Osipenko, Mathematical Notes 19(1), 29-40 (1976)

2. J. Garnett, Bounded analytic functions (Mir, Moscow, 1984)

3. P. Kusis, Introduction to the theory of $H^{p}$ spaces (Mir, Moscow, 1984)

4. C. Micchelli, T. Rivlin, Lect. Notes 9, 21-93 (1982)

5. T.J. Rivlin, Contemp. Math. 9, 121-151 (1982)

6. S.D. Fisher, C.A. Micchelli, Duke Math J. 47(4), 789-801 (1980)

7. K.Yu. Osipenko, M.I. Stesin, Mathematical Notes 49(4), 95-104 (1991)

8. R.R. Akopyan, Mathematical notes. 99(2), 163-170 (2016). DOI: 10.4213/mzm1074.1

9. G.G. Magaril-Ilyaev, Izv. RAS. Series: Mathematics. 78(6), 83-102. (2014). DOI: 10.4213/im8182

10. R.R. Akopyan, Izv. RAS. Series: Mathematics, 210(10), 1348. (2019)

11. R.R. Akopyan, Mathematical Notes, 99(2), 163-170 (2016). Doi: https//doi.org/10.4213/mzm10741

12. R.R. Akopyan, Tr. IMM UB RAS, 25(2), 21-29 (2019). Doi: 10.21538/0134-48892019-25-2-21-29

13. M.P. Ovchintsev, E3S Web of Conferences, 110, 01043 (2019) https://doi.org/10.1051/e3sconf/201911001043

14. S.Ya. Khavinson, Fundamentals of the theory of extremal problems for bounded analytic functions and their various generalizations (Moscow, Moscow Institute of Civil Engineering named after V.V. Kuybyshev (MISI), 1981)

15. W.W. Rogosinski, H. Schapiro, Acta Math. 90(3), 287-318 (1954) 